\title{
Sirolimus in patients with clinically active systemic lupus erythematosus resistant to, or intolerant of, conventional medications: a single-arm, open-label, phase 1/2 trial
}

\author{
Zhi-Wei Lai, MD, Ryan Kelly, PhD, Thomas Winans, BSc, Ivan Marchena, MD, Ashwini \\ Shadakshari, MD, Julie Yu, MD, Maha Dawood, MD, Ricardo Garcia, MD, Hajra Tily, MD, Lisa \\ Francis, MD, Prof. Stephen V Faraone, PhD, Prof. Paul E Phillips, MD, and Prof. Andras \\ Perl, MD \\ Division of Rheumatology (Prof A Perl MD, Z-W Lai MD, R Kelly PhD, T Winans BSc, I Marchena \\ MD, A Shadakshari MD, J Yu MD, M Dawood MD, R Garcia MD, H Tily MD, L Francis MD, Prof P \\ E Phillips MD), Department of Psychiatry (Prof S V Faraone PhD), Department of Medicine (Prof \\ A Perl, Z-W Lai, R Kelly, T Winans, I Marchena, A Shadakshari, J Yu, M Dawood, R Garcia, H \\ Tily, L Francis, Prof P E Phillips), Department of Microbiology and Immunology (Prof A Perl), and \\ Department of Biochemistry and Molecular Biology (Prof A Perl), State University of New York \\ Upstate Medical University, Syracuse, NY, USA
}

\section{Summary}

Background-Patients with systemic lupus erythematosus have T-cell dysfunction that has been attributed to the activation of the mammalian target of rapamycin (mTOR). Rapamycin inhibits antigen-induced T-cell proliferation and has been developed as a medication under the generic designation of sirolimus. We assessed safety, tolerance, and efficacy of sirolimus in a prospective, biomarker-driven, open-label clinical trial.

\begin{abstract}
Methods-We did a single-arm, open-label, phase 1/2 trial of sirolimus in patients with active systemic lupus erythematosus disease unresponsive to, or intolerant of, conventional medications at the State University of New York Upstate Medical University (Syracuse, NY, USA). Eligible participants (aged $\geq 18$ years) had active systemic lupus erythematosus fulfilling four or more of 11 diagnostic criteria defined by the American College of Rheumatology. We excluded patients with allergy or intolerance to sirolimus, patients with life-threatening manifestations of systemic lupus erythematosus, proteinuria, a urine protein to creatinine ratio higher than $0 \cdot 5$, anaemia, leucopenia, or thrombocytopenia. Patients received oral sirolimus at a starting dose of $2 \mathrm{mg}$ per day, with dose adjusted according to tolerance and to maintain a therapeutic range of $6-15 \mathrm{ng} / \mathrm{mL}$. Patients were treated with sirolimus for 12 months. Safety outcomes included tolerance as assessed by the occurrence of common side-effects. The primary efficacy endpoint was decrease in disease
\end{abstract}

\footnotetext{
Correspondence to: Prof Andras Perl, Department of Medicine, State University of Upstate Medical University New York, Syracuse, NY 13210, USA, perla@upstate.edu.

Contributors

AP conceived and designed the study. All authors were involved in data collection. Z-WL, SVF, and AP analysed and interpreted the data. All authors were involved in development, review, and approval of the manuscript.

Declaration of interests

We declare no competing interests.
} 
activity, assessed using the British Isles Lupus Assessment Group (BILAG) index and the Systemic Lupus Erythematosus Disease Activity Index (SLEDAI). Blood samples of 56 matched healthy individuals were obtained as controls for immunobiological outcomes monitored at each visit. The primary efficacy endpoint was assessed in all patients who completed 12 months of treatment, and all patients who received at least one dose of treatment were included in the safety analyses. This trial is registered with ClinicalTrials.gov, number NCT00779194.

Findings-Between March 9, 2009, and Dec 8, 2014, 43 patients were enrolled, three of whom did not meet eligibility criteria. 11 of the 40 eligible patients discontinued study treatment because of intolerance $(n=2)$ or non-compliance $(n=9)$. SLEDAI and BILAG disease activity scores were reduced during 12 months of treatment in $16(55 \%)$ of 29 patients who completed treatment. Mean SLEDAI score decreased from 10.2 (SD 5.6) at enrolment to 4.8 (4.5) after 12 months of treatment $(\mathrm{p}<0.001)$ and the mean total BILAG index score decreased from $28.4(12.4)$ at enrolment to 17.4 (10.7) after 12 months of treatment ( $<<0.001)$. The mean daily dose of prednisone required to control disease activity decreased from $23.7 \mathrm{mg}$ (SD 9.6) to $7.2 \mathrm{mg} \mathrm{(2.3;} \mathrm{p<0.001)} \mathrm{after} 12$ months of treatment. Sirolimus expanded $\mathrm{CD} 4^{+} \mathrm{CD} 25^{+} \mathrm{FoxP}^{+}$regulatory $\mathrm{T}$ cells and $\mathrm{CD} 8^{+}$memory T-cell populations and inhibited interleukin- 4 and interleukin- 17 production by $\mathrm{CD} 4^{+}$and $\mathrm{CD}^{-} \mathrm{CD}^{-}$ double-negative T cells after 12 months. CD8 ${ }^{+}$memory $\mathrm{T}$ cells were selectively expanded in SRIresponders. Patient liver function and lymphocyte counts were unchanged. Although HDLcholesterol $(Z=-2 \cdot 50, \mathrm{p}=0 \cdot 012)$, neutrophil counts $(Z=-1 \cdot 92, \mathrm{p}=0.054)$, and haemoglobin $(Z=$ $-2 \cdot 83, \mathrm{p}=0.005)$ were moderately reduced during treatment, all changes occurred within a range that was considered safe. Platelet counts were slightly elevated during treatment $(Z=2 \cdot 06$, $\mathrm{p}=0 \cdot 0400)$.

Interpretation-These data show that a progressive improvement in disease activity is associated with correction of pro-inflammatory T-cell lineage specification in patients with active systemic lupus erythematosus during 12 months of sirolimus treatment. Follow-up placebo-controlled clinical trials in diverse patient populations are warranted to further define the role of mTOR blockade in treatment of systemic lupus erythematosus.

\section{Introduction}

Systemic lupus erythematosus is a chronic inflammatory disease that primarily affects women of child-bearing age, with debilitating and potentially life-threatening consequences.

${ }^{1}$ The disease represents an unmet medical need because the drugs that are available are only partly effective and have considerable side-effects. ${ }^{2}$ Consequently, $10 \%$ of patients with systemic lupus erythematosus die within 5 years of diagnosis. ${ }^{3}$ Although the cause of systemic lupus erythematosus is incompletely understood, it is thought to involve cellular dysfunction of the immune system and the production of autoantibodies. ${ }^{1}$ Activation of the mechanistic target of rapamycin (mTOR) has emerged as a key driver of abnormal lineage specification within the immune system, ${ }^{4}$ which has been attributed to metabolic stress in people with systemic lupus erythematosus. ${ }^{5-8}$ Although mTOR activation ${ }^{9}$ and its therapeutic reversal were originally identified in the $\mathrm{T}$ cells of patients with systemic lupus erythematosus, ${ }^{10}$ studies have also reported mTOR activation in parenchymal organs such as the liver in mice ${ }^{11}$ and the kidneys in patients with antiphospholipid syndrome. ${ }^{12}$ 
mTOR is a serine-threonine kinase that takes its name after rapamycin, an antifungal antibiotic produced by Streptomyces hygroscopicus, a soil bacterium from Easter Island, known by its inhabitants as Rapa Nui. ${ }^{13}$ Rapamycin effectively inhibits antigen-induced Tcell proliferation, ${ }^{14}$ and has been developed as a medication to prevent organ transplant rejection under the generic designation of sirolimus. ${ }^{15}$ Sirolimus forms a high-affinity complex with its cellular receptor, FKBP12, a $12 \mathrm{kD}$ protein that is overexpressed in lupus $\mathrm{T}$ cells. ${ }^{9}$ This complex of sirolimus and FKBP12 blocks mTOR activation. ${ }^{16}$ In vivo, sirolimus completely abrogated autoimmunity in lupus-prone mice, ${ }^{17,18}$ and blocked disease activity in a retrospective study of patients with systemic lupus erythematosus. ${ }^{10} \mathrm{We}$ initiated this prospective study to assess tolerance, safety, and the metabolic, immunological, and therapeutic effect of sirolimus in patients with severe systemic lupus erythematosus who are intolerant of, or do not respond to, other conventional medications.

\section{Methods}

\section{Study design and participants}

We did a prospective, single-arm, open-label, phase 1/2 trial at the Division of Rheumatology, State University of New York Upstate Medical University (Syracuse, NY, USA) with approval from the institutional review board and the US Food and Drug Administration (IND 101566). The study protocol is available online.

We assessed the safety and efficacy of sirolimus for patients with active systemic lupus erythematosus. We also obtained blood from a healthy control group matched with patients for age, sex, and ethnicity, and freshly isolated cells from healthy control participants were used in parallel as controls for immunological studies (appendix pp 3-9).

We enrolled patients aged 18 years or older with systemic lupus erythematosus that fulfilled four or more of 11 diagnostic criteria defined by the American College of Rheumatology. 19,20 We anticipated that most patients enrolled in the study would have active disease (Systemic Lupus Erythematosus Disease Activity Index [SLEDAI] score $\geq 4)^{21}$ and were receiving prednisone $10 \mathrm{mg}$ or more per day, but these were not formal inclusion criteria. Patients with allergy or intolerance to sirolimus, and patients with life-threatening manifestations of systemic lupus erythematosus (eg, cerebritis substantiated by inflammatory MRI lesions, catastrophic antiphospholipid antibody syndrome, rapid progressive glomerulo-nephritis requiring intravenous cyclophosphamide, or a glomerular filtration rate of $<40 \mathrm{~mL} / \mathrm{min}$ ) were excluded. Patients with proteinuria exceeding $500 \mathrm{mg} / 24$ $\mathrm{h}$, a urine protein to creatinine ratio higher than $0 \cdot 5$, anaemia (haemoglobin $<10 \mathrm{~g} / \mathrm{dL}$ ), leucopenia (white blood cell [WBC] count $<3000$ cells per $\mu \mathrm{L}$ ), or thrombocytopenia (platelet count $<100000$ cells per $\mu \mathrm{L}$ ) were excluded. Patients with a WBC count of 3000 3500 per $\mu \mathrm{L}$, haemoglobin concentration of $10-12 \mathrm{~g} / \mathrm{dL}$, or platelet counts of 100 000-150 000 per $\mu \mathrm{L}$ were monitored every week for 1 month. If $\mathrm{WBC}$ and platelet counts were sustained or improved, patients were followed up according to standard protocol. Patients with total cholesterol concentrations of more than $300 \mathrm{mg} / \mathrm{dL}$ or triglyceride concentrations of more than $400 \mathrm{mg} / \mathrm{dL}$ were also excluded. Patients who were pregnant were excluded and use of contraceptives was required in potentially fertile female patients. Patients who developed pneumonitis that was confirmed by high-resolution $\mathrm{CT}^{22}$ were excluded. Patients 
with acute infection requiring antibiotics were not to be enrolled. Written informed consent was obtained from each patient and matched healthy control participant using forms approved by the institutional review board of the State University of New York Upstate Medical University.

\section{Procedures}

Patients had a complete physical examination before enrolment. Patients received oral sirolimus, at a starting dose of $2 \mathrm{mg}$ daily, which was adjusted on the basis of tolerance and to maintain a therapeutic range of 6-15 ng/mL. Patients were treated with sirolimus for 12 months. During the study, the prednisone dose was titrated to control disease activity and was monitored throughout the trial.

Patients taking hydroxychloroquine and existing immunosuppressive medications, such as mycophenolate mofetil, could continue treatment, but the dose was adjusted or discontinued during the trial. With the exception of sirolimus, no new immunosuppressive drugs were initiated during the trial.

Clinical and laboratory assessments were done on day 0 (before administration of the first sirolimus dose; visit 1), and 1 month (visit 2), 3 months (visit 3), 6 months (visit 4), 9 months (visit 5), and 12 months after initiation of sirolimus treatment (visit 6). Sirolimus levels and complete blood counts were also measured on days 15 and 60. Patients had physical examinations of the cardiovascular, respiratory, gastrointestinal, musculoskeletal, and neurological systems, and the skin, head, neck, sinuses, and nasal and oral cavities at each visit. Routine blood tests were also done at each visit, which included complete blood counts, liver and kidney function tests, fasting lipid profile, urinalysis, and laboratory tests, such as anti-double-stranded DNA, C3, and C4. All immunobiological assessments are described in the appendix (pp 5,6). Treatment was discontinued in patients with decreased WBC or platelet counts at any weekly follow-up and in patients who developed infections and required intravenous antibiotics and had no clinical improvement within 5 days.

Common side-effects (eg, nausea, headache, mouth sores) reported in a previous trial $^{23}$ were specifically assessed at each visit and reviewed by the data and safety monitoring board.

\section{Outcomes}

Safety outcomes included tolerance as assessed by the occurrence of common side-effects. The development of non-healing oral ulcers or new onset headache indicated intolerance to sirolimus. Hyperlipidaemia, thrombocytopenia, mucositis, oedema, and proteinuria, which have been observed in renal transplant patients, ${ }^{24}$ were also monitored as safety outcomes.

The primary efficacy endpoint was decrease in disease activity, defined as a decrease in SLEDAI and BILAG scores at each visit during treatment (months 1-12) compared with baseline.

Secondary endpoints were prednisone dose required to control disease activity, and changes in immunobiological biomarkers of clinical responsiveness compared against healthy controls. Since the initiation of our trial, a consensus has emerged that clinical efficacy 
should be determined by comparing response rates between study groups after at least 1 year of intervention, ${ }^{25}$ which is advocated by the US Food and Drug Administration for phase 3 trials seeking regulatory approval. ${ }^{26}$

Therefore, we determined the response to sirolimus treatment as defined by the Systemic Lupus Erythematosus Responder Index (SRI), for which we used a composite of the SLEDAI and BILAG scores. Physician Global Assessment (PGA) scores were not recorded because the original SLEDAI scoring system was used, ${ }^{21}$ and therefore PGA scores were not used in the calculation of the SRI in this study. A responder was defined as having at least a 4 point decrease in the SLEDAI score from baseline and an absence of BILAG A or two BILAG B scores. ${ }^{27}$

We also looked at the individual components of the SLEDAI and BILAG scores, including the proportion of patients who had a disease flare (defined as a having either one BILAG A score or two BILAG B scores across all organ systems).

\section{Statistical analysis}

Power and sample size requirements were based on a type I error rate of 0.05 , two-tailed $t$ tests, and a minimum power level of 0.80, using SPSS statistical software (version 17.0). Effect size estimates were based on our preliminary data ${ }^{10}$ and the relevant literature ${ }^{28,29}$ was used to compare mean SLEDAI and BILAG scores after a meaningful duration of intervention (ie, 12 months). The primary efficacy endpoint was assessed in all patients who completed 12 months of treatment, and all patients who received at least one dose of treatment were included in the safety analyses. Repeated measures mixed model logistic regression analysis, $\chi^{2}$ tests, and two-tailed paired $t$ tests were used to assess the effects of sirolimus on clinical indices and biomarkers recorded at visits $2-6$ compared with visit 1 and $\mathrm{p}<0.05$ was considered to indicate a statistically significant difference. Patients and controls were compared using mixed-effects models and two-tailed unpaired $t$ tests. Two-tailed $\chi^{2}$ and Fisher's exact tests were used to compare categorical parameters with GraphPad Prism software (version 5.0). To analyse the data obtained for all repeated measures from each patient, we used a mixed-effects model approach with study visit as a fixed effect and patient identification number as a random effect, using Stata software (version 15.0). This model uses all available datapoints and assumes that missing values are missing at random. For group comparisons, we included the main effect of the group and the group-by-visit interaction as fixed effects. The interaction tests whether the change across visits differs between groups. The group comparison models used matched pairs of patients; pairs were included as a random effect. We used a Gaussian model, with the exception of ordinal variables for which we used ordinal logistic regression. All dependent variables that were percentages were transformed into logits using the following equation: $\operatorname{logit}(\mathrm{x})=\ln (\mathrm{x} /(1-\mathrm{x}))$. When no $Z$ value was reported, mixed-effects models could not converge to yield an analysis report. The Stata software package reports changes as $Z$ values with two decimal places and $p$ values up to three decimal places. $p$-values from Stata generated as $p=0.000$ were reported as $p<0.001$. Area under the receiver operating characteristic curve (AUC) logistic regression analysis was done with Metaboanalyst 3.0. ${ }^{30}$ Observations and complaints regarding drug 
intolerance, adverse events, and serious adverse events were documented according to the data and safety monitoring plan.

This study is registered with ClinicalTrials.gov, number NCT00779194.

\section{Role of the funding source}

The funders of the study had no role in study design, data collection, data analysis, data interpretation, or writing of the report. ZL and AP had full access to all the data in the study and had final responsibility for the decision to submit for publication.

\section{Results}

Between March 9, 2009, and Dec 8, 2014, 43 patients and 56 matched healthy controls were enrolled. Three consented patients (patients 38,41 , and 42) did not meet eligibility criteria after screening. The mean age was 45.4 years (SD 14.3) in the patient group and 45.4 years (12.7) in the control group (appendix p 11). 38 patients were women, 35 of whom were white and three of whom were African American. Two patients were men, both of whom were white. Baseline clinical characteristics of all enrolled patients, including age, sex, ethnicity, SLEDAI score, BILAG index score, prednisone dose, and medication use are shown in the table. 51 controls were women, including 45 white individuals, five AfricanAmerican individuals, and one Hispanic individual. The five male controls were white.

29 patients completed 12 months of treatment. $11(28 \%)$ of 40 eligible patients discontinued study treatment because of intolerance $(n=2)$ or non-compliance $(n=9)$. Of the nine patients excluded because of non-compliance, one patient moved away and eight patients did not comply with study protocol.

Sirolimus concentrations were targeted within the therapeutic range of $6-15 \mathrm{ng} / \mathrm{mL}$ in all patients. Mean concentrations increased from less than $2 \mathrm{ng} / \mathrm{mL}$ before treatment (visit 1) to $7.7 \mathrm{ng} / \mathrm{mL}(3.7)$ at visit $6(Z=4.70, \mathrm{p}<0.001$; paired $t \mathrm{p}=0.0037$; figure $1 \mathrm{~A})$. Mean haemoglobin concentrations $(Z=-2 \cdot 83, \mathrm{p}=0 \cdot 005$; figure $1 \mathrm{~B})$ were reduced following 2 months of treatment with sirolimus; however, values remained within a range that was considered to be safe. Total WBC count $(Z=-1.92, \mathrm{p}=0.055$; figure $1 \mathrm{C})$, and neutrophil count $(Z=-1.92, \mathrm{p}=0 \cdot 054$; figure 1D) were reduced, but not significantly so. Although no significant difference in mean lymphocyte count was found between visit 1 and visit 6 $(Z=1.03, \mathrm{p}=0.301$; figure $1 \mathrm{E})$, a moderate increase in mean platelet count was observed $(Z=2 \cdot 06, \mathrm{p}=0 \cdot 0400$; figure $1 \mathrm{~F})$. Liver function, assessed by aspartate aminotransferase and alanine aminotransferase concentrations, was not affected (data not shown). Although components of fasting lipid panels were transiently altered at visits 2,3 , and 4 within the windows of tolerance, no statistically significant differences were observed at visits 5 and 6 (appendix p 13). However, mixed model logistic regression analysis revealed a significant reduction in HDL $(Z=-2 \cdot 50, \mathrm{p}=0 \cdot 012)$ and a significant increase in the non-HDL lipoprotein fraction $(Z=1.97, \mathrm{p}=0.049)$ during treatment. No significant differences were identified in the concentrations of total cholesterol $(Z=0.47, \mathrm{p}=0 \cdot 636)$, LDL ( $Z=1 \cdot 67, \mathrm{p}=0 \cdot 096)$, VLDL $(Z=0 \cdot 85, \mathrm{p}=0 \cdot 396)$, or triglycerides $(Z=0 \cdot 72, \mathrm{p}=0 \cdot 471)$ compared with baseline. One patient 
developed extensive oral ulcers after 12 weeks of treatment with sirolimus, which resolved after discontinuation of study treatment.

The mean SLEDAI score decreased from 10.2 (SD 5.6) at visit 1 to $4.8(4 \cdot 5)$ at visit 6 $(\mathrm{p}<0.001$; figure 2A). The total BILAG index score decreased from a mean of 28.4 (SD $12.4)$ at visit 1 to $17.4(10 \cdot 7)$ at visit $6(\mathrm{p}<0.001$; figure $2 \mathrm{~B})$. Using a mixed model logistic regression approach to analyse all repeated measures from each patient, mean SLEDAI ( $Z=$ $-6 \cdot 06, \mathrm{p}<0 \cdot 001)$ and BILAG $(Z=-4 \cdot 76, \mathrm{p}<0.001)$ scores were both significantly reduced during 12 months of treatment with sirolimus in 16 [55\%] of 29 patients. When correcting for multiple comparisons, each component outcome had a p value of less than 0.025.

Mean daily prednisone dose required to control disease activity was reduced from $23.7 \mathrm{mg}$ (SD 9.6) at visit 1 to $7 \cdot 2 \mathrm{mg}(2 \cdot 3)$ at visit $6(Z=-5 \cdot 24, \mathrm{p}<0 \cdot 001$, figure $2 \mathrm{C})$.

Because our study had no placebo group, we compared the number of patients who met responder criteria according to the SRI at visit 6 with that at visit 2 using visit 1 as a reference. Four (17\%) of 24 patients met criteria for the SRI at visit 2 compared with 19 $(66 \%)$ of 29 patients at visit $6(\mathrm{p}<0 \cdot 001$; figure $2 \mathrm{D})$.

The proportion of patients who had a flare of active lupus was reduced significantly compared with visit 1 after 3 months of treatment (figure 3A). The proportion of patients who scored 3 points or more in the mucocutaneous (figure 3B) and musculoskeletal (figure 3C) BILAG organ domains was significantly reduced during sirolimus treatment. Within these organ domain scores, the prevalence of maculopapular eruptions (mild), malar erythema, arthritis, and arthralgia were reduced (figure 3). The proportion of patients who scored 3 or more did not significantly reduce during treatment for any of the other BILAG organ domains (appendix p 14). Cardiovascular and pulmonary domain scores (figure 3D) did not significantly improve during treatment. Mean mucocutaneous (figure 3E), musculoskeletal (figure 3F), cardiopulmonary (figure 3G), and vasculitis (figure 3H) BILAG scores were reduced during 12 months of treatment. Regarding SLEDAI components, the prevalence of arthritis (figure 3I), new rash (figure 3J), pyuria (figure 3K), and hypocomplementaemia (figure $3 \mathrm{~L}$ ) were reduced during treatment with sirolimus. No significant reductions were reported for the other 20 individual components of the SLEDAI score.

At visit 1, six (15\%) of 40 patients had anti-DNA antibodies compared with two (7\%) of 29 patients at visit 6 ; however, this difference was not statistically significant $(\mathrm{p}=0.4529)$.

We assessed the mean proportions of specific lymphocyte subsets associated with clinical improvement in disease activity during 12 months of treatment. At baseline, patients with systemic lupus erythematosus had a significantly higher mean proportion of $\mathrm{CD} 8^{+} \mathrm{CD} 45 \mathrm{RA}^{+}$ naive $\mathrm{T}$ cells and a lower proportion of $\mathrm{CD} 8^{+} \mathrm{CD} 45 \mathrm{RO}^{+}$memory $\mathrm{T}$ cells than did matched healthy controls (figure 4; appendix p 15). These differences were found in freshly isolated and in-vitro stimulated $\mathrm{T}$ cells. The expansion of naive T-cell populations and depletion of $\mathrm{CD} 8^{+}$memory $\mathrm{T}$ cells were improved during sirolimus treatment (figure 4; appendix $\mathrm{p} 15$ ). Expansion of CD8 memory T-cell populations was confined to the 11 patients with clinical improvement (SLEDAI score decreased by $\_4$ points) at 12 months of treatment (appendix $p$ 
15). Although CD4 memory T-cell populations were not significantly depleted in patients with systemic lupus erythematosus, their CD4 memory T-cell counts were increased among patients who had a decrease of 4 points or more in SLEDAI scores (appendix $\mathrm{p} 15$ ). Depletion of $\mathrm{CD}^{+}$memory $\mathrm{T}$ cells during sirolimus treatment mainly involved the $\mathrm{CD}^{-} 2 \mathrm{~L}^{-}$ CD197- effector-memory T cells (figure 5A) and, to a lesser extent, CD62L ${ }^{+} \mathrm{CD} 197^{+}$ central-memory compartments ${ }^{31}$ (figure 5B). Immunophenotyping effectively distinguished SRI responders from non-responders (figure 5C). Depletion of CD62L ${ }^{-} \mathrm{CD} 197^{-}$effectormemory $\mathrm{CD} 8^{+} \mathrm{T}$ cells emerged as the strongest predictor of therapeutic response to sirolimus with an AUC value of 0.967 (figure 5D).

A representative patient-control pair (appendix p 17) and cumulative analysis (appendix $p$ 18) showed that patients with systemic lupus erythematosus had FoxP $3^{+}$regulatory T-cell depletion that appeared to reverse during sirolimus treatment in vivo. $\mathrm{CD} 4^{+} \mathrm{FoxP} 3^{+} \mathrm{T}$ regulatory cells were significantly expanded in patients with systemic lupus erythematosus at visit 6 compared with baseline ( $Z=4 \cdot 09, \mathrm{p}<0 \cdot 001$; appendix p 18).

Pro-inflammatory T-cell lineage specification was characterised by increased production of interleukin 4 and interleukin 17 by $\mathrm{CD} 4$ and $\mathrm{CD}^{+}{ }^{+} \mathrm{CD} 4^{-} \mathrm{CD} 8^{-}$double-negative $\mathrm{T}$ cells, which was reduced during 12 months of treatment with sirolimus (appendix p 19).

Interleukin-17 production was also increased in CD8 lupus T cells, and reduced during sirolimus treatment (appendix p 19). Interferon- $\gamma$ production increased during sirolimus treatment in $\mathrm{CD}^{+}$and double negative $\mathrm{T}$ cells (appendix $\mathrm{p} 19$ ). Mean mitochondrial mass in double-negative T cells - an important source of oxidative stress in systemic lupus erythematosus ${ }^{32}$ - was higher in patients with systemic lupus erythematosus than healthy controls at baseline, and seemed to reduce during sirolimus treatment (appendix p 20).

Antiphospholipid antibodies, such as anti- $\beta_{2}$ glycoprotein I and anti-cardiolipin antibodies can cause considerable comorbidities (ie, antiphospholipid syndrome) in patients with and without systemic lupus erythematosus. ${ }^{33}$ IgM and IgA anti- $\beta_{2}$ glycoprotein I and anticardiolipin antibody production had reduced after 1 month of treatment, and this reduction was sustained at 12 months of treatment (appendix p 21). IgG anti- $\beta_{2}$ glycoprotein I and anti-cardiolipin antibodies were not affected during sirolimus treatment (data not shown).

\section{Discussion}

This mechanistic trial provides preliminary evidence that sirolimus is safe, well tolerated, and clinically efficacious in patients with systemic lupus erythematosus when carefully monitored for reversible oral ulcers, headaches, and cytopenia. Disease activity, as assessed via the reversal of pro-inflammatory T-cell lineage specification, showed improvement at visit 6 (after 12 months of sirolimus treatment).

Among side-effects commonly reported in renal transplant recipients treated with sirolimus, ${ }^{24}$ haemoglobin and neutrophil counts were reduced; however, values remained within the safe range. By contrast to patients who have transplants, platelet counts were moderately increased, which is attributed to thrombocytopenia being a manifestation of lupus disease activity. Transient hyperlipidaemia was observed in patients during the first 6 months of 
treatment. Although hyperlipidaemia is a comorbidity of systemic lupus erythematosus, ${ }^{34}$ and normalisation of this disorder at 12 months of treatment might represent an outcome of clinical efficacy, monitoring appears to be indicated within individual patients and in future clinical trials.

Importantly, disease activity was reduced during sirolimus treatment in patients who were intolerant of, or unresponsive to, other immunosuppressant medications. BILAG scores were improved after 1 month of treatment with sirolimus, and continued to improve during the 12 month treatment period, supporting the clinical efficacy of sirolimus. SLEDAI scores decreased after 3 months of treatment and continued to decline during the 12 month treatment period. The SRI response rate after 12 months of treatment (66\%) is similar to that for belimumab (58\%), ${ }^{35}$ which is the only medication that has been approved for systemic lupus erythematosus by the US Food and Drug Administration since the 1960s. As of Sept 12, 2017, 18 of 19 SRIresponsive patients remained on sirolimus treatment. The longest duration of treatment with sirolimus in our cohort is 15 years.

In this study, a marked expansion of $\mathrm{CD} 8{ }^{+} \mathrm{CD} 45 \mathrm{RA}^{+}$naive $\mathrm{T}$ cells and contraction of $\mathrm{CD} 8{ }^{+} \mathrm{CD} 45 \mathrm{RO}^{+}$memory $\mathrm{T}$ cells was found in patients with systemic lupus erythematosus at baseline compared with matched healthy controls. Depletion of $\mathrm{CD} 8^{+}$memory $\mathrm{T}$ cells, which primarily affects the effector-memory compartment, was corrected during treatment with sirolimus for 12 months. CD4 memory T-cell populations also expanded during sirolimus treatment, but only among SRI responders. Depletion of CD8 effector-memory T cells compromises T-cell responses to viruses that are considered triggers of disease flares in patients with systemic lupus erythematosus. ${ }^{36}$ Thus, expansion of CD8 effector-memory T cells by sirolimus might block virally induced disease activation ${ }^{37}$ via type I interferon production. ${ }^{38}$

At baseline, regulatory T-cell populations were depleted in patients with systemic lupus erythematosus compared with healthy controls, which was progressively reversed during 12 months of sirolimus treatment. Pro-inflammatory T-cell lineage specification also involves expansion of interleukin-4-producing T-helper (Th)-2 cells and interleukin-17-producing Th17 cells and depletion of interferon- $\boldsymbol{\gamma}$-producing Th1 cells, all of which were moderated during treatment with sirolimus for 12 months. Mitochondrial mass appeared to reduce in double-negative $\mathrm{T}$ cells during treatment with sirolimus, which drives oxidative stress and pro-inflammatory necrotic death in systemic lupus erythematosus. ${ }^{39,40}$ Importantly, blockade of mitochondrial oxidative stress with acetylcysteine has also been shown to abrogate mTOR activation and had clinical efficacy in a double-blind placebo-controlled trial. ${ }^{41}$ These findings are consistent with a positive feedback loop between metabolic stress and mTOR activation in lupus T cells. ${ }^{4}$

$\operatorname{IgM}$ and $\operatorname{Ig} \mathrm{A}$ antiphospholipid antibody production was reduced after 1 month of sirolimus treatment, which was sustained for 12 months. In a retrospective study ${ }^{12}$ of antiphospholipid syndrome nephropathy, seven of ten patients treated with sirolimus had a functioning renal allograft 144 months after transplantation compared with only three of 27 patients not treated with sirolimus. Diagnosis of systemic lupus erythematosus, antiphospholipid syndrome, or antiphospholipid antibody production has not been reported in patients who 
benefited from sirolimus. ${ }^{12}$ In addition to findings in lupus-prone mice, ${ }^{11}$ these results further substantiate the hypothesis that mTOR blockade might benefit patients with antiphospholipid syndrome who otherwise require life-long anticoagulation. ${ }^{33}$

Our study had several limitations, including the open-label design and that patients were only recruited at a single centre. We used modified SRI criteria, ${ }^{42}$ which included a decrease of 4 points or more in SLEDAI scores and absence of BILAG A or two BILAG B scores, but not Physicians Global Assessment scores. 35 of the 40 patients enrolled were white women, therefore the results might primarily represent clinical efficacy in this patient group. Moreover, most patients had active disease, were unresponsive or intolerant to conventional medications, and received high-dose steroid therapy. Thus, sirolimus might not be as beneficial in patients with milder disease.

The safety and therapeutic efficacy of sirolimus are attributed to the involvement of mTOR in lupus pathogenesis. mTOR is activated before disease onset in lupus-prone mice ${ }^{11}$ and before flares in patients with systemic lupus erythematosus. ${ }^{40}$ Moreover, genetic hyperactivity of mTOR has been associated with concurrent systemic lupus erythematosus. ${ }^{43}$ In the present study, two patients discontinued treatment with sirolimus before 12 months because of intolerance. The overall safety and clinical efficacy of sirolimus in systemic lupus erythematosus, and its association with the therapeutic reversal of pro-inflammatory lineage specification (ie, depletion of $\mathrm{T}$ regulatory cells and CD8 effector-memory $\mathrm{T}$ cells and expansion of interleukin-4 and interleukin-17-producing $\mathrm{CD}^{+} \mathrm{CD}^{-}{ }^{-} \mathrm{CD} 8^{-}$doublenegative $\mathrm{T}$ cells), which are newly delineated through treatment of human beings in vivo, are strengths of the study. However, the open-label approach and having enrolled only three AfricanAmerican patients, who typically have more severe disease, are limitations of the study. Therefore, follow-up placebo-controlled clinical trials in more diverse patient populations are warranted to define the role of mTOR blockade in treatment of systemic lupus erythematosus.

\section{Supplementary Material}

Refer to Web version on PubMed Central for supplementary material.

\section{Acknowledgments}

Funding Pfizer, the National Institutes of Health, and the Central New York Community Foundation.

This work was supported in part by an investigator-initiated research grant from Pfizer (P0468X1-4470/ WS1234172) and grants from the National Institutes of Health (AI 048079, AI 072648, and AI 122176) and the Central New York Community Foundation. We thank Melissa Reale for dispensing the study drug and excellent record keeping.

\section{References}

1. Tsokos GC. Systemic lupus erythematosus. N Engl J Med. 2011; 365:2110-21. [PubMed: 22129255]

2. Francis L, Perl A. Pharmacotherapy of systemic lupus erythematosus. Expert Opin Pharmacother. 2009; 10:1481-94. [PubMed: 19505215] 
3. Pons-Estel GJ, Alarcon GS, Scofield L, Reinlib L, Cooper GS. Understanding the epidemiology and progression of systemic lupus erythematosus. Semin Arthritis Rheum. 2010; 39:257-68. [PubMed: 19136143]

4. Perl A. Mechanistic target of rapamycin pathway activation in rheumatic diseases. Nat Rev Rheumatol. 2016; 12:169-82. [PubMed: 26698023]

5. Perl A, Hancko R, Lai Z-W, et al. Comprehensive metabolome analyses reveal N-acetylcysteineresponsive accumulation of kynurenine in systemic lupus erythematosus: implications for activation of the mechanistic target of rapamycin. Metabolomics. 2015; 11:1157-74. [PubMed: 26366134]

6. Chi H. Regulation and function of mTOR signalling in T cell fate decisions. Nat Rev Immunol. 2012; 12:325-38. [PubMed: 22517423]

7. Morel L. Immunometabolism in systemic lupus erythematosus. Nat Rev Rheumatol. 2017; 13:280 90. [PubMed: 28360423]

8. Bettencourt IA, Powell JD. Targeting metabolism as a novel therapeutic approach to autoimmunity, inflammation, and transplantation. J Immunol. 2017; 198:999-1005. [PubMed: 28115589]

9. Fernandez DR, Telarico T, Bonilla E, et al. Activation of mTOR controls the loss of TCR in lupus T cells through HRES-1/Rab4-regulated lysosomal degradation. J Immunol. 2009; 182:2063-73.

[PubMed: 19201859]

10. Fernandez D, Bonilla E, Mirza N, Niland B, Perl A. Sirolimus reduces disease activity and normalizes T-cell activation-induced calcium fluxing in patients with systemic lupus erythematosus. Arthritis Rheum. 2006; 54:2983-88. [PubMed: 16947529]

11. Oaks Z, Winans T, Caza T, et al. Mitochondrial dysfunction in the liver and antiphospholipid antibody production precede disease onset and respond to rapamycin in lupus-prone mice. Arthritis Rheumatol. 2016; 68:2728-39. [PubMed: 27332042]

12. Canaud G, Bienaimé F, Tabarin F, et al. Inhibition of the mTORC pathway in the antiphospholipid syndrome. N Engl J Med. 2014; 371:303-12. [PubMed: 25054716]

13. Vézina C, Kudelski A, Sehgal SN. Sirolimus (AY 22,989), a new antifungal antibiotic. I. Taxonomy of the producing streptomycete and isolation of the active principle. J Antibiot. 1975; 28:721-26. [PubMed: 1102508]

14. Sehgal SN, Bansback CC. Rapamycin: in vitro profile of a new immunosuppressive macrolide. Ann NY Acad Sci. 1993; 685:58-67. [PubMed: 8363266]

15. Collier DSJ, Calne R, Thiru S, et al. Rapamycin in experimental renal allografts in dogs and pigs. Transplant Proc. 1990; 22:1674-75. [PubMed: 2143858]

16. Sabatini DM, Erdjument-Bromage H, Lui M, Tempst P, Snyder SH. RAFT1: a mammalian protein that binds to FKBP12 in a rapamycin-dependent fashion and is homologous to yeast TORs. Cell. 1994; 78:35-43. [PubMed: 7518356]

17. Warner LM, Adams LM, Sehgal SN. Rapamycin prolongs survival and arrests pathophysiologic changes in murine systemic lupus erythematosus. Arthritis Rheum. 1994; 37:289-97. [PubMed: 8129783]

18. Caza TN, Fernandez D, Talaber G, et al. HRES-1/RAB4-mediated depletion of DRP1 impairs mitochondrial homeostasis and represents a target for treatment in SLE. Ann Rheum Dis. 2014; 73:1887-97.

19. Tan EM, Cohen AS, Fries JF, et al. The 1982 revised criteria for the classification of systemic lupus erythematosus. Arthritis Rheum. 1982; 25:1271-77. [PubMed: 7138600]

20. Hochberg MC. Updating the American College of Rheumatology revised criteria for the classification of systemic lupus erythematosus. Arthritis Rheum. 1997; 40:1725.

21. Bombardier C, Gladman DD, Urowitz MB, Caron D, Chang CH. the committee on prognosis studies in SLE. Derivation of the SLEDAI. A disease activity index for lupus patients. Arth Rheum. 1992; 35:630-40. [PubMed: 1599520]

22. Champion L, Stern M, Israël-Biet D, et al. Brief communication: sirolimus-associated pneumonitis: 24 cases in renal transplant recipients. Ann Intern Med. 2006; 144:505-09. [PubMed: 16585664]

23. El-Chemaly S, Taveira-Dasilva A, Goldberg HJ, et al. Sirolimus and autophagy inhibition in lymphangioleiomyomatosis: results of a phase I clinical trial. Chest. 2017; 151:1302-10.

[PubMed: 28192114] 
24. Ponticelli C. The pros and the cons of mTOR inhibitors in kidney transplantation. Exp Rev Clin Immunol. 2013; 10:295-305.

25. Strand V, Chu AD. Measuring outcomes in systemic lupus erythematosus clinical trials. Exp Rev Pharmacoecon Outcomes Res. 2011; 11:455-68.

26. US Department of Health and Human Services, Food and Drug Administration. [accessed Feb 21, 2018] Guidance for Industry. Systemic lupus erythematosus - developing medical products for treatment. http://www.fda.gov/downloads/Drugs/GuidanceComplianceRegulatoryInformation/ Guidances/ucm072063.pdf

27. Isenberg DA, Rahman A, Allen E, et al. BILAG 2004. Development and initial validation of an updated version of the British Isles Lupus Assessment Group's disease activity index for patients with systemic lupus erythematosus. Rheumatology. 2005; 44:902-06. [PubMed: 15814577]

28. Yee CS, Farewell VT, Isenberg DA, et al. The use of Systemic Lupus Erythematosus Disease Activity Index-2000 to define active disease and minimal clinically meaningful change based on data from a large cohort of systemic lupus erythematosus patients. Rheumatology. 2011; 50:98288. [PubMed: 21245073]

29. Brunner HI, Higgins GC, Klein-Gitelman MS, et al. Minimal clinically important differences of disease activity indices in childhood-onset systemic lupus erythematosus. Arthritis Care Res. 2010; 62:950-99.

30. Xia J, Wishart DS. Using MetaboAnalyst 3.0 for comprehensive metabolomics data analysis. Curr Protoc Bioinform. 2016; 55:14.10.1-14.10.91.

31. Sallusto F, Lenig D, Forster R, Lipp M, Lanzavecchia A. Two subsets of memory T lymphocytes with distinct homing potentials and effector functions. Nature. 1999; 401:708-12. [PubMed: 10537110]

32. Perl A. Oxidative stress in the pathology and treatment of systemic lupus erythematosus. Nat Rev Rheumatol. 2013; 9:674-86. [PubMed: 24100461]

33. Lockshin MD, Erkan D. Treatment of the antiphospholipid syndrome. N Engl J Med. 2003; 349:1177-79. [PubMed: 13679533]

34. Tselios K, Koumaras C, Gladman DD, Urowitz MB. Dyslipidemia in systemic lupus erythematosus: just another comorbidity? Semin Arthritis Rheum. 2016; 45:604-10. [PubMed: 26711309]

35. Navarra SV, Guzman RM, Gallacher AE, et al. Efficacy and safety of belimumab in patients with active systemic lupus erythematosus: a randomised, placebo-controlled, phase 3 trial. Lancet. 2011; 377:721-31. [PubMed: 21296403]

36. Comte D, Karampetsou MP, Yoshida N, Kis-Toth K, Kyttaris VC, Tsokos GC. Signaling lymphocytic activation molecule family member 7 engagement restores defective effector $\mathrm{CD} 8+\mathrm{T}$ cell function in systemic lupus erythematosus. Arthritis Rheum. 2017; 69:1035-44.

37. Perl A. Mechanisms of viral pathogenesis in rheumatic disease. Ann Rheum Dis. 1999; 58:454-61. [PubMed: 10419862]

38. Kirou KA, Lee C, George S, Louca K, Peterson MG, Crow MK. Activation of the interferon-alpha pathway identifies a subgroup of systemic lupus erythematosus patients with distinct serologic features and active disease. Arthritis Rheum. 2005; 52:1491-503. [PubMed: 15880830]

39. Gergely PJ, Grossman C, Niland B, et al. Mitochondrial hyperpolarization and ATP depletion in patients with systemic lupus erythematosus. Arthritis Rheum. 2002; 46:175-90. [PubMed: 11817589]

40. Lai Z-W, Borsuk R, Shadakshari A, et al. mTOR activation triggers IL-4 production and necrotic death of double-negative $\mathrm{T}$ cells in patients with systemic lupus erythematosus. J Immunol. 2013; 191:2236-46. [PubMed: 23913957]

41. Lai Z-W, Hanczko R, Bonilla E, et al. N-acetylcysteine reduces disease activity by blocking mTOR in T cells of lupus patients. Arthritis Rheum. 2012; 64:2937-46. [PubMed: 22549432]

42. Furie RA, Petri MA, Wallace DJ, et al. Novel evidence-based systemic lupus erythematosus responder index. Arthritis Care Res. 2009; 61:1143-51.

43. Oaks Z, Winans T, Huang N, Banki K, Perl A. Activation of the mechanistic target of rapamycin in SLE: explosion of evidence in the last five years. Curr Rheum Rep. 2016; 18:73. 


\section{Research in context}

\section{Evidence before this study}

An unmet need exists for treatment in systemic lupus erythematosus. Mammalian target of rapamycin (mTOR) activation has been identified as a driver of pro-inflammatory lineage skewing in the immune system. We searched PubMed for English language articles published between Jan 1, 2000, and Oct 13, 2017, using the search terms "sirolimus," "rapamycin," or "mTOR" and "lupus". We found that research investigating the blockade of mTOR by sirolimus has been limited to a single retrospective study and two confirmatory case reports, which suggest that sirolimus has clinical efficacy in patients with systemic lupus erythematosus.

\section{Added value of this study}

This is the first prospective study of sirolimus in patients with systemic lupus erythematosus. This open-label study shows that treatment with sirolimus is safe, with rapid and lasting improvement of disease activity after 12 months of treatment resulting from the blockade of pro-inflammatory T-cell lineage specification in patients with active systemic lupus erythematosus. The depletion of CD8 effector-memory T cells predicts therapeutic response, and the progressive restoration of this cell population occurs with clinical improvement.

\section{Implications of all the available evidence}

The results of this study show that sirolimus is potentially a safe and efficacious treatment in patients with active systemic lupus erythematosus who are unresponsive to, or intolerant of, conventional medications. The results warrant double-blind placebocontrolled follow-up studies with sirolimus alone or in combination with potentially synergistic interventions, such as acetylcysteine, in larger and more diverse patient populations. 

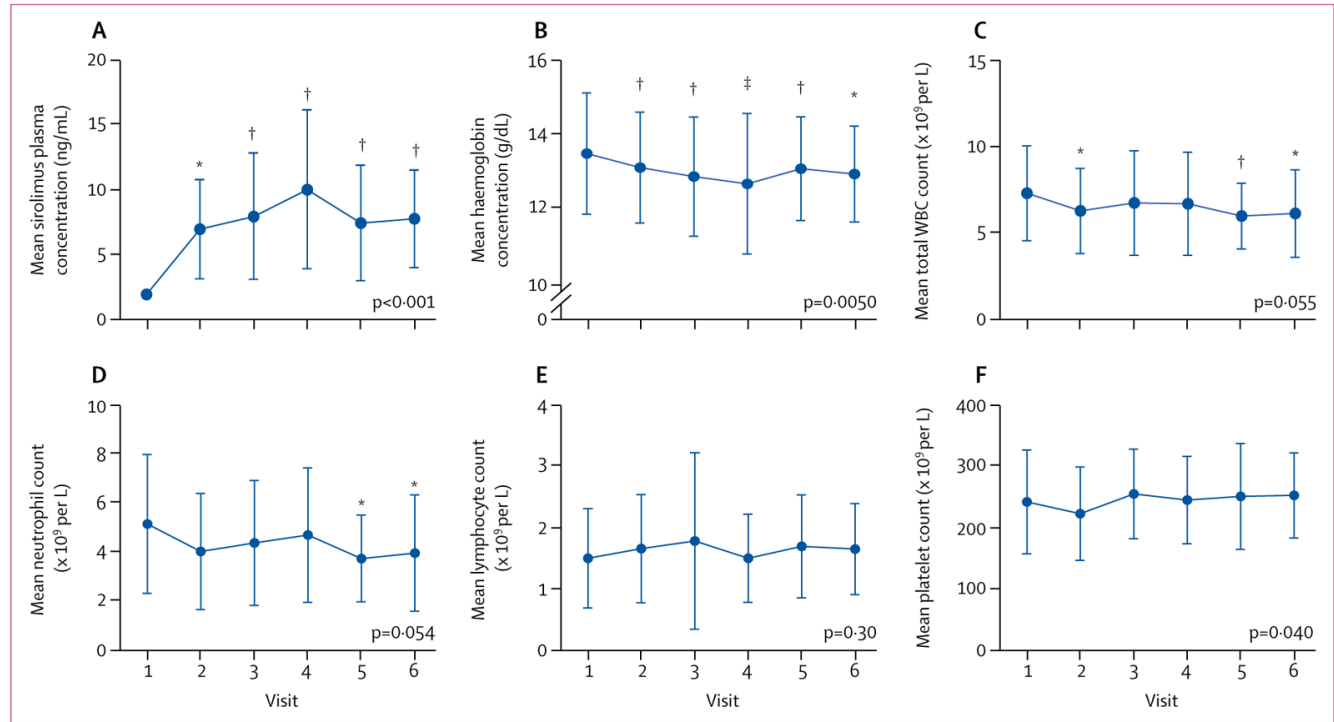

Figure 1. Safety outcomes

Mean sirolimus plasma concentration (A), mean haemoglobin concentration (B), mean total WBC count (C), and mean neutrophil (D), lymphocyte (E), and platelet counts (F) were measured before treatment (visit 1) and after initiation of treatment at 1 month (visit 2), 3 months (visit 3), 6 months (visit 4), 9 months (visit 5), and 12 months (visit 6) in 40 patients with systemic lupus erythmatosus. Overall changes in safety endpoints during treatment were assessed by repeated measures analysis using a mixed-effects model logistic regression approach with exact $\mathrm{p}$ values indicated for each safety outcome. Changes in safety endpoints at each visit (visits 2-6) were assessed by two-tailed paired $t$ tests relative to visit 1 . Error bars show SD. WBC=white blood cell. *p<0.05. $\dagger \mathrm{p}<0 \cdot 01 . \neq \mathrm{p}<0 \cdot 001$. 

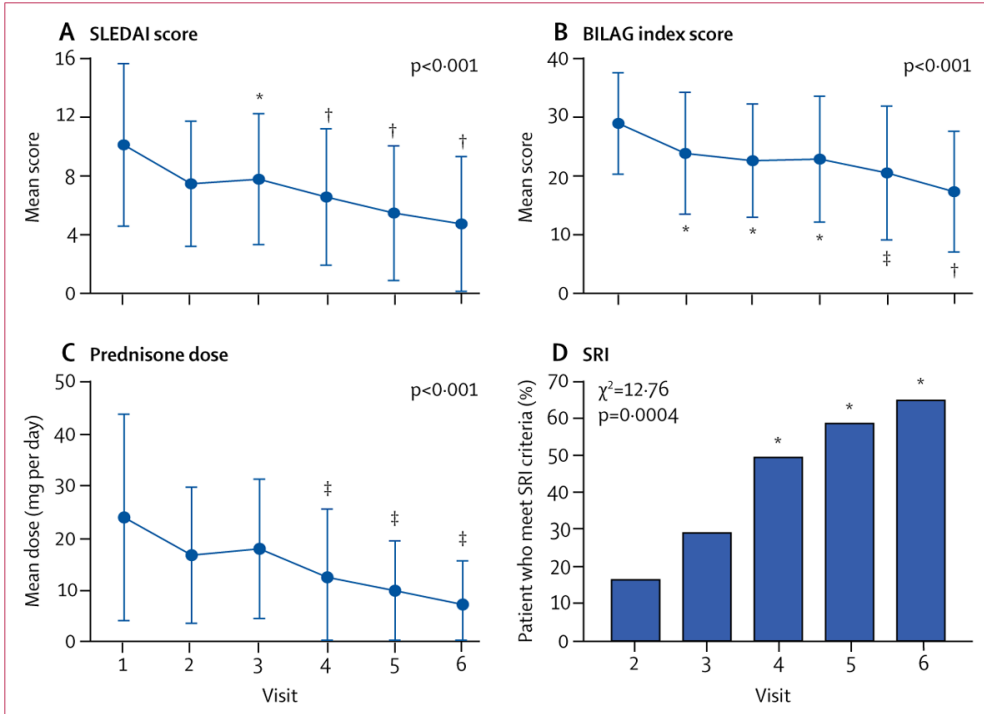

Figure 2. Clinical efficacy outcomes

Mean SLEDAI score (A), BILAG index score (B), and daily prednisone dose (C) at baseline (visit 1) and during treatment (visits 2-6). Overall changes in SLEDAI, BILAG, and prednisone dosage during sirolimus treatment were assessed by repeated measures analysis using a mixed model logistic regression approach with exact $\mathrm{p}$ values indicated for each outcome. Changes in SLEDAI, BILAG, and prednisone dosage at each visit (visits 2-6) were also assessed by two-tailed paired $t$ test relative to visit 1 . (D) The proportion of patients who met responder criteria according to the SRI at visits 3-6 were compared with that at visit 2 using visit 1 as a reference point. Overall distribution of responders and nonresponders for SRI at visits 2-6 were also assessed by two-tailed $\chi^{2}$ test. Error bars show SD. SLEDAI=Systemic Lupus Erythematosus Disease Activity Index. BILAG=British Isles Lupus Assessment Group. SRI=Systemic Lupus Erythematosus Responder Index. *p<0·01. $\uparrow \mathrm{p}<0 \cdot 001 . \neq \mathrm{p}<0 \cdot 05$. 

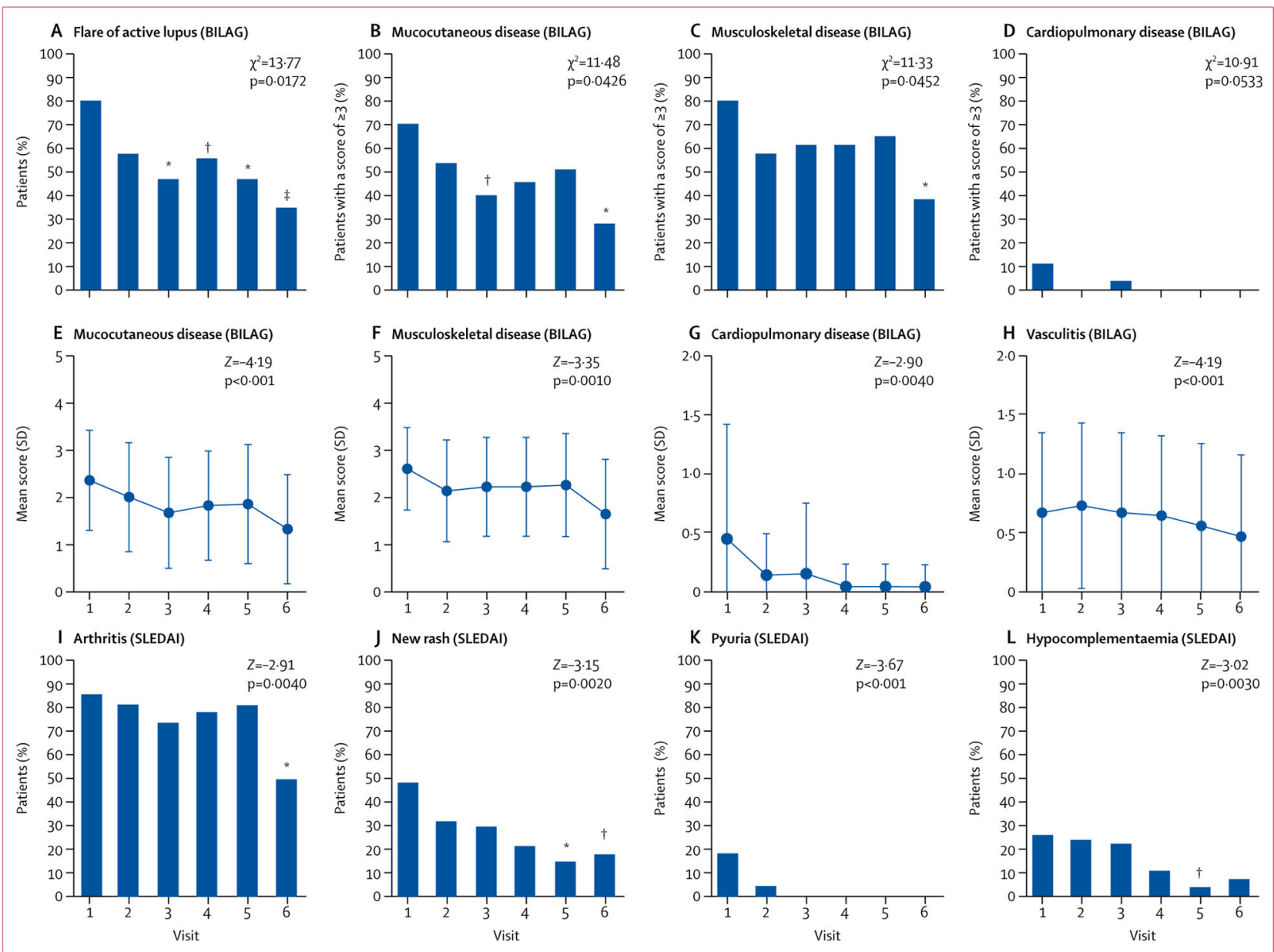

Figure 3. BILAG-defined disease flares and selected BILAG organ domain scores and SLEDAI component scores

Proportion of patients with a flare of active lupus (a new BILAG A or two new BILAG B scores in at least one organ system; A) and a BILAG organ domain score of 3 or more for mucocutaneous disease (B), musculoskeletal disease (C) and cardiovascular and pulmonary disease (D). Mean BILAG organ domain scores for mucocutaneous disease (E), musculoskeletal disease $(F)$, cardiovascular and pulmonary disease $(\mathrm{G})$, and vasculitis $(\mathrm{H})$. Mean SLEDAI score for arthritis (I), new rash (J), pyuria (K), and hypocomplementaemia (L). Scores were assessed relative to day of enrolment (before treatment, visit 1) and after treatment for 1 month (visit 2), 3 months (visit 3), 6 months (visit 4), 9 months (visit 5), and 12 months (visit 6). Effects of sirolimus were assessed by two-tailed paired $t$ tests relative to visit 1. Overall distribution of the proportion of patients with a BILAG-defined flare (A), proportion of patients with an organ domain score of 3 or more (B-D), and overall distribution of SLEDAI components (I-L) were also assessed by $\chi^{2}$ test. Mixed model logistic regression approach was used to analyse effect ( $Z$-values) using repeated measures from each patient. Error bars show SD. BILAG=British Isles Lupus Assessment Group. SLEDAI $=$ Systemic Lupus Erythematosus Disease Activity Index. ${ }^{*} \mathrm{p}<0 \cdot 01 . \dagger \mathrm{p}<0 \cdot 05$. $\ddagger \mathrm{p}<0 \cdot 001$. 

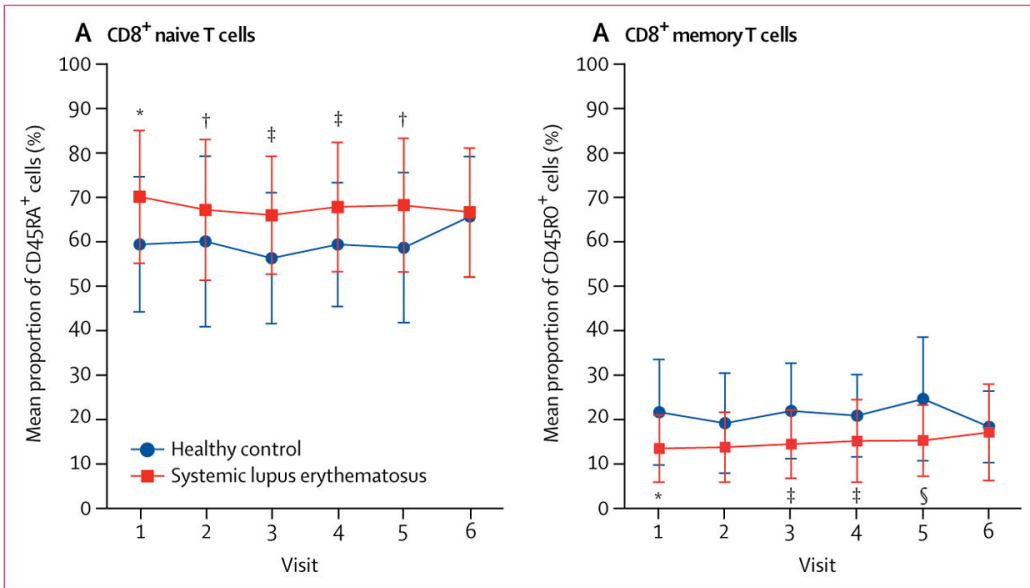

Figure 4. Changes in naive $\mathrm{CD8} \mathrm{T}$ cell and memory $\mathrm{CD8} \mathrm{T}$ cells populations Changes in expansion of naive CD8 T cells (A) and changes in memory CD8 T cells (B), as shown in fresh cells. Changes in the proportion of naive and memory CD8 T cells at each visit were assessed by two-tailed unpaired $t$ tests relative to matched healthy controls at each timepoint and two-tailed paired $t$ tests relative to visit 1 . Error bars show SD. The number of patients (and healthy controls) contributing data differed at each visit. ${ }^{*} \mathrm{p}<0.01$ relative to healthy matched control.

$\dagger \mathrm{p}<0.05$ relative to visit $1 . \ddagger \mathrm{p}<0.05$ relative to healthy matched control. $\$ \mathrm{p}<0.001$ relative to healthy matched control. 

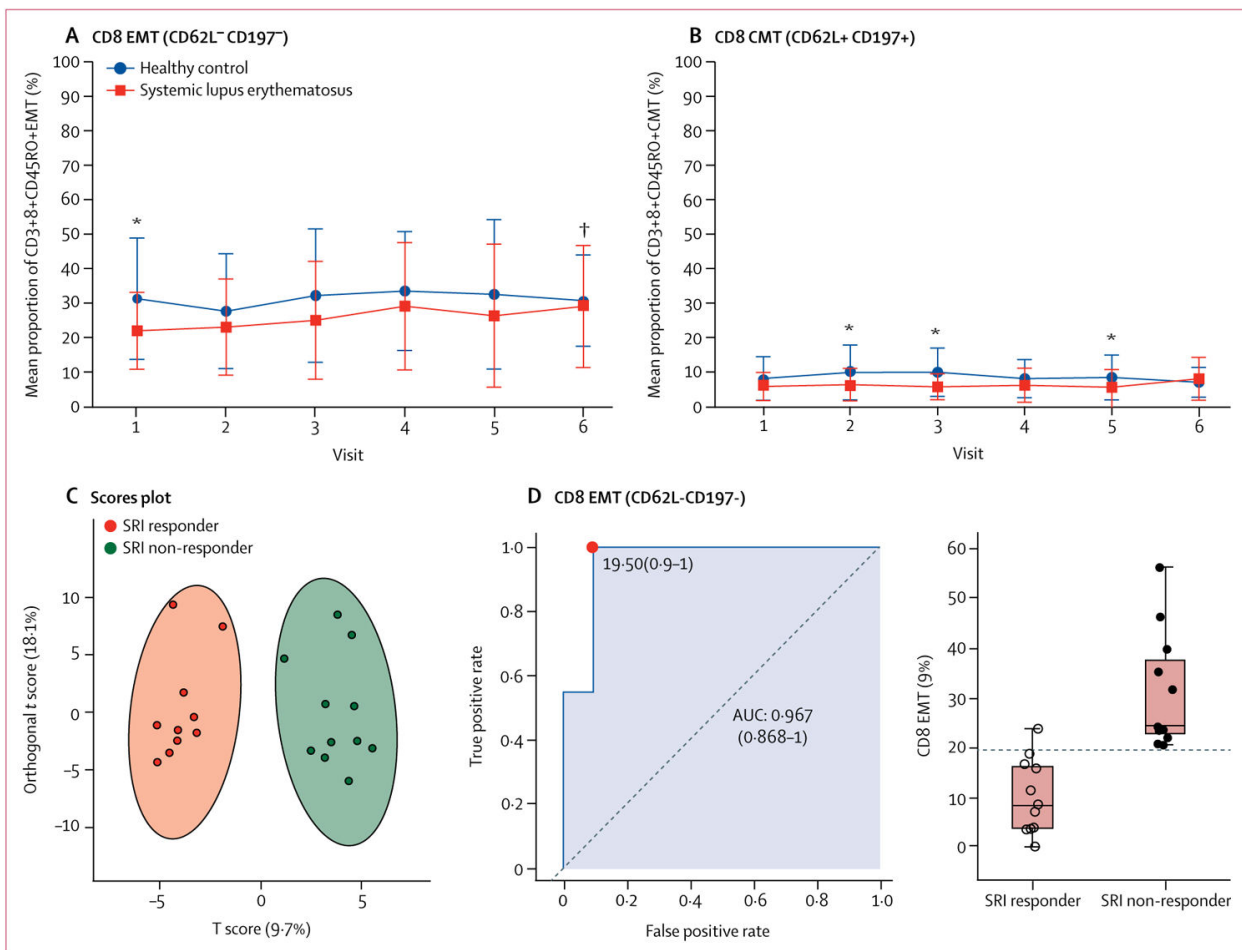

Figure 5. Changes in CD8 EMT and CMT cells

Changes in $\mathrm{CD}^{+} \mathrm{CD}^{-} \mathrm{L}^{-} \mathrm{CD} 197^{-} \mathrm{EMT}$ cells (A) and $\mathrm{CD}^{+} \mathrm{CD}^{-} 2 \mathrm{~L}^{+} \mathrm{CD} 197^{+} \mathrm{CMT}$ cells (B) during sirolimus treatment, compared with healthy controls. Changes in CD8 EMT and CMT cells were assessed by two-tailed unpaired $t$ test relative to matched healthy controls and by two-tailed paired $t$ tests relative to visit 1 in each patient. Error bars show SD. (C) Orthogonal partial least squares discriminant analysis of 22 patients, for whom a complete dataset was available, shows discrimination of SRI responsive and SRI non-responsive patients by two components of immunobiological biomarkers, using complete biomarker datasets. (D) Discrimination of SRI responsive and SRI non-responsive patients on the basis of the AUC logistic regression approach. The left panel shows the AUC CI, true positive and false positive rates, and CI. The right panel shows the abundance of EMT cells in SRI responsive and SRI non-responsive patients. Black horizontal lines indicate mean values in each patient group. Vertical lines indicate range. The horizontal dotted line shows the optimum cutoff between responsive and non-responsive patients. EMT=effector memory T. $\mathrm{CMT}=$ central memory $\mathrm{T}$. SRI=systemic lupus erythematosus responder index. AUC=area under the receiver operating characteristic curve. ${ }^{*} \mathrm{p}<0.05$ relative to matched healthy controls. $\nmid \mathrm{p}<0.05$ relative to visit 1 in each patient. 


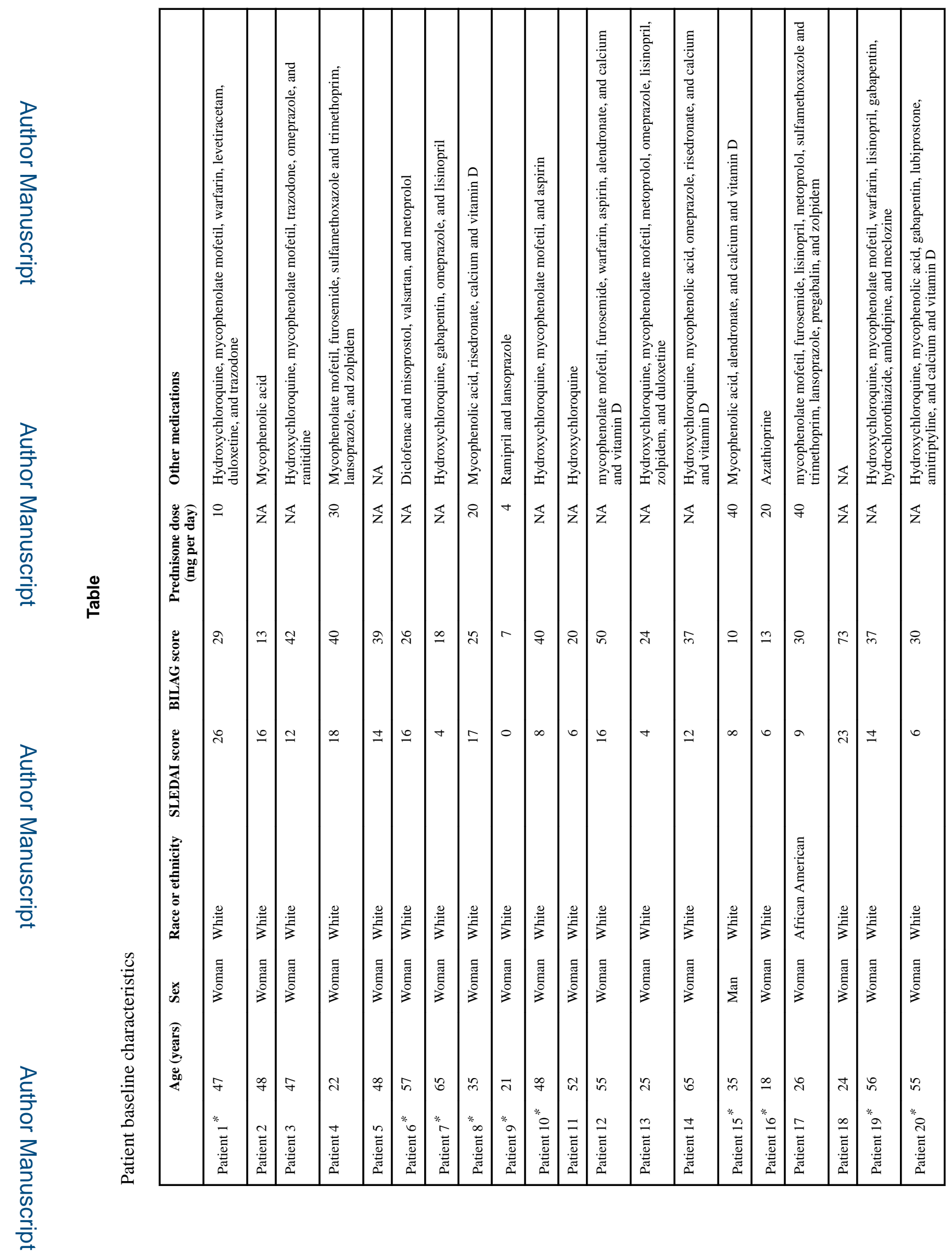

Lancet. Author manuscript; available in PMC 2019 March 24. 


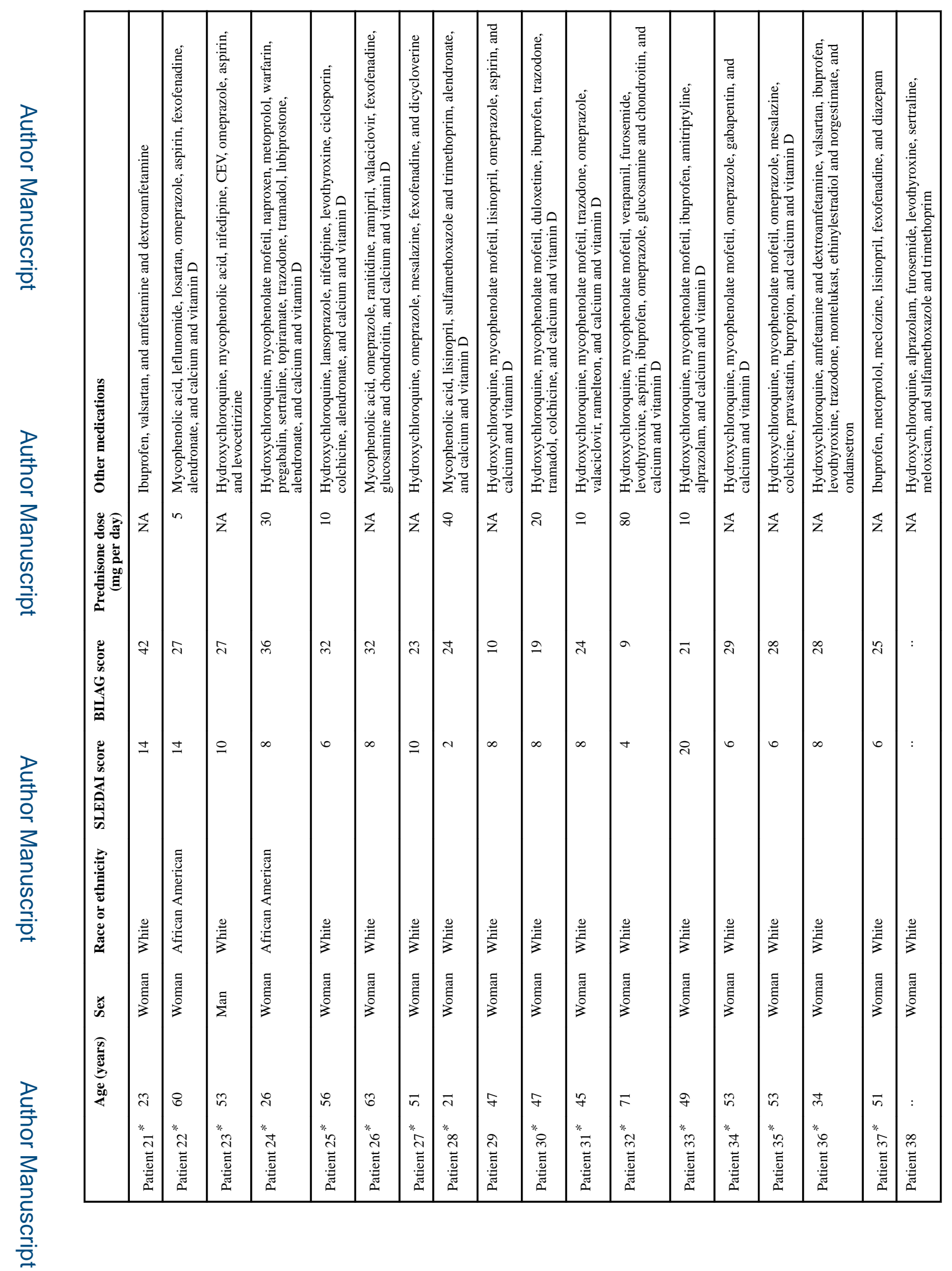

Lancet. Author manuscript; available in PMC 2019 March 24. 


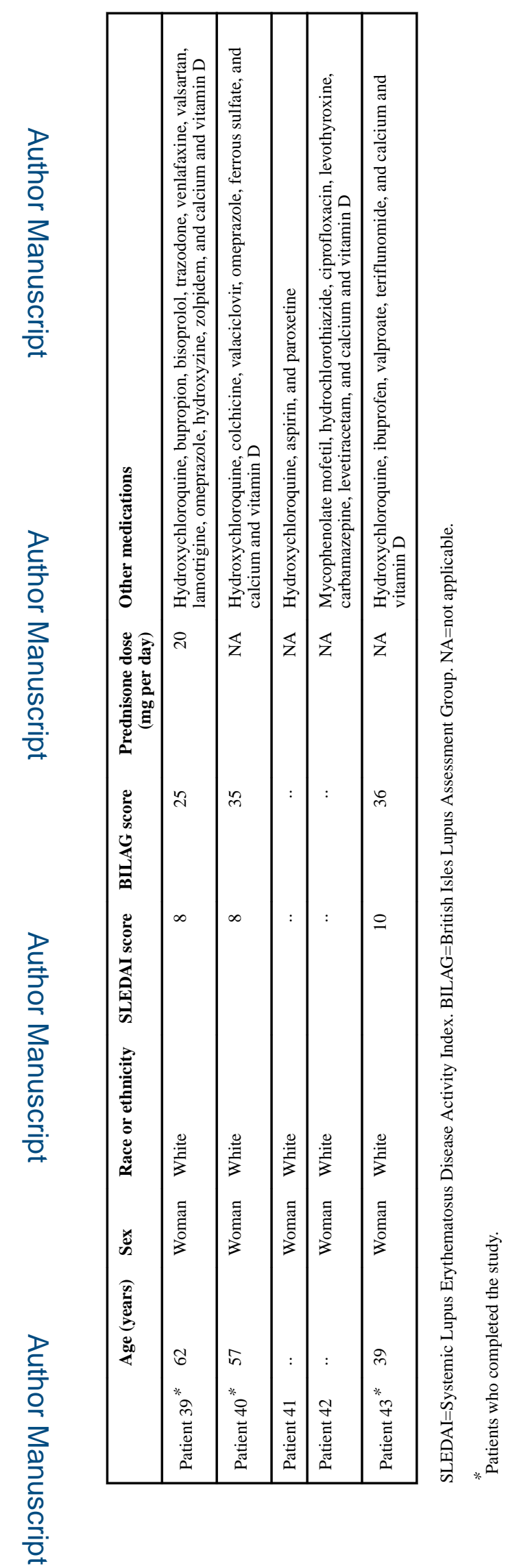

Lancet. Author manuscript; available in PMC 2019 March 24. 